\title{
A narrative review of plaque and brain imaging biomarkers for stroke risk stratification in patients with atherosclerotic carotid artery disease
}

\author{
Simone J. A. Donners, Raechel J. Toorop, Dominique P. V. de Kleijn, Gert J. de Borst \\ Department of Vascular Surgery, Division of Surgical Specialties, University Medical Center Utrecht, Utrecht University, Utrecht, The Netherlands \\ Contributions: (I) Conception and design: GJ de Borst, SJA Donners; (II) Administrative support: None; (III) Provision of study materials or patients: \\ All authors; (IV) Collection and assembly of data: SJA Donners; (V) Data analysis and interpretation: All authors; (VI) Manuscript writing: All \\ authors; (VII) Final approval of manuscript: All authors. \\ Correspondence to: Gert J. de Borst, MD, PhD. Department of Vascular surgery G04.129, UMC Utrecht, Heidelberglaan 100, 3584 CX Utrecht, The \\ Netherlands. Email: G.J.deBorst-2@umcutrecht.nl.
}

Objective: In this narrative review, we aim to review imaging biomarkers that carry the potential to noninvasively guide stroke risk stratification for treatment optimization.

Background: Carotid atherosclerosis plays a fundamental part in the occurrence of ischemic stroke. International guidelines select the optimal treatment strategy still mainly based on the presence of clinical symptoms and the degree of stenosis for stroke prevention in patients with atherosclerotic carotid plaques. These guidelines, based on randomized controlled trials that were conducted three decades ago, recommend carotid revascularization in symptomatic patients with high degree of stenosis versus a conservative approach for most asymptomatic patients. Due to optimization of best medical therapy and risk factor control, it is suggested that a subgroup of symptomatic patients is at lower risk of stroke and may not benefit from revascularization, whereas a selective subgroup of high-risk asymptomatic patients would benefit from this procedure.

Methods: A literature search was performed for articles published up to December 2020 using PubMed, EMBASE and Scopus. Based on the literature found, change in stenosis degree and volume, plaque echolucency, plaque surface, intraplaque haemorrhage, lipid-rich necrotic core, thin fibrous cap, inflammation, neovascularization, microembolic signals, cerebrovascular reserve, intracranial collaterals, silent brain infarcts, diffusion weighted imaging lesions and white matters lesions have the potential to predict stroke risk.

Conclusions: The applicability of imaging biomarkers needs to be further improved before the potential synergistic prognostic ability of imaging biomarkers can be verified on top of the clinical biomarkers. In the future, the routine and combined assessment of both plaque and brain imaging biomarkers might help to improve optimization of treatment strategies in individual patients with atherosclerotic carotid artery disease.

Keywords: Carotid artery disease; stroke risk; carotid endarterectomy (CEA); plaque imaging; brain imaging

Submitted Mar 12, 2021. Accepted for publication May 20, 2021.

doi: $10.21037 / \mathrm{atm}-21-1166$

View this article at: https://dx.doi.org/10.21037/atm-21-1166 


\section{Introduction}

Stroke affects approximately 12 million people worldwide each year, making it the second leading cause of death (1). Atherosclerosis of the carotid artery is a common cause of stroke and causes besides death enormous morbidity with long-term disability and financial burden. Patients with atherosclerotic carotid artery stenosis presenting with cerebral ischemic events [transient ischemic attack (TIA) or stroke] in the recent six months are classified as symptomatic, and asymptomatic when having no cerebral ischemic events in the past six months. Randomized controlled trials (RCT) conducted over three decades ago have demonstrated that carotid endarterectomy (CEA) effectively reduced the risk of future stroke in both patients with symptomatic and, to lesser extent, in patients with asymptomatic significant carotid artery atherosclerotic stenosis (2-4). Patient selection in these RCTs was primarily based on a history of prior cerebral ischemic events and degree of carotid stenosis on catheter-based angiography. Thirty years later, while carotid artery stenting (CAS) has emerged as a less invasive alternative, the presence of neurological symptoms and degree of stenosis still form the fundament of clinical decision making for the individual patient $(5,6)$. Long-term results of the landmark trials have shown that the number needed to treat (NNT) to prevent one stroke is six for symptomatic patients and 22 for asymptomatic patients $(7,8)$. Due to improvements in best medical therapy (BMT) these NNTs have further increased despite a parallel trend in decline in periprocedural adverse events (9). As such, the optimal treatment strategy for patients with asymptomatic high-grade stenosis has become increasingly controversial since the low riskbenefit ratio between revascularization procedures and medical treatment. While in patients with asymptomatic carotid disease the most relevant question is 'who benefits most from surgical intervention?', the main question in symptomatic patients for long time accounted 'what is the best timing for surgical intervention?'. Symptomatic patients are at high risk for recurrent stroke especially in the early time after the index event (10). New markers are needed to prioritize patients in whom urgent or expedited CEA or CAS will provide most benefit. Controversially, the European Carotid Surgery Trial 2 (ECST-2) aimed to discuss whether, based on clinical and plaque characteristics, a subgroup of symptomatic patients could be identified that would be better off with optimal medication instead of revascularization for long term stroke prevention.
More recently, a prospective cohort study showed that symptomatic patients with a non-significant $(<50 \%)$ carotid stenosis also have a substantial risk of $7.4 \%$ on recurrent ipsilateral stroke at 3 years (11). These findings highlight the strong need to extent the assessment of atherosclerotic carotid artery disease beyond clinical symptomatic status and imaging-based degree of stenosis for optimization in management strategies.

In order to identify patients with atherosclerotic carotid plaques with a so called 'high risk' for future or recurrent stroke, plaque and brain characteristics gained a lot of attention. Postmortem studies have attempted to correlate several ex vivo plaque characteristics [e.g., high infiltration of inflammatory cells, large lipid-rich necrotic cores (LRNC) and a thin fibrous cap (FC)] with rupture prone plaques (12), whereas biobank plaque studies attempted to correlate these high risk plaque characteristics with predicted stroke risk (13). Extensive developments in imaging techniques [e.g., ultrasound (US), computed tomography (CT) and magnetic resonance imaging (MRI)] have enabled to identify high risk plaques with plaque and brain imaging features. The contributive value of these in vivo imaging features on the natural course of atherosclerotic carotid disease and the subsequent risk of future and recurrent ischemic events needs further validation (14). A recent meta-analysis reported that the presence of 'high risk' plaque imaging features is associated with an annual risk of $4.3 \%$ on ipsilateral ischemic stroke in asymptomatic patients, far exceeding the commonly accepted annual rate of $1 \%(15)$. Also, patients with mild carotid plaques $(<50 \%)$ presenting with cryptogenic stroke or embolic stroke of undetermined source show significant higher amount of complicated carotid plaques on the ipsilateral side than the contralateral side, suggesting an association between clinical symptoms and ipsilateral complicated plaques (16). The 2017 European Society for Vascular Surgery (ESVS) Guidelines suggest that in vivo imaging of the plaque and brain can non-invasively identify patients with a deemed higher risk for future or recurrent stroke while being under optimal medical treatment (5). All suggested imaging based predictive parameters, however, were derived from individual post-hoc analyses, and should be validated first before being implemented in daily clinical practice.

Contemporary reviews focused on imaging biomarkers of solely asymptomatic or symptomatic carotid stenosis patients, or defined only plaque imaging or brain imaging biomarkers. This review will give a comprehensive overview of imaging biomarkers of the carotid plaque and brain 


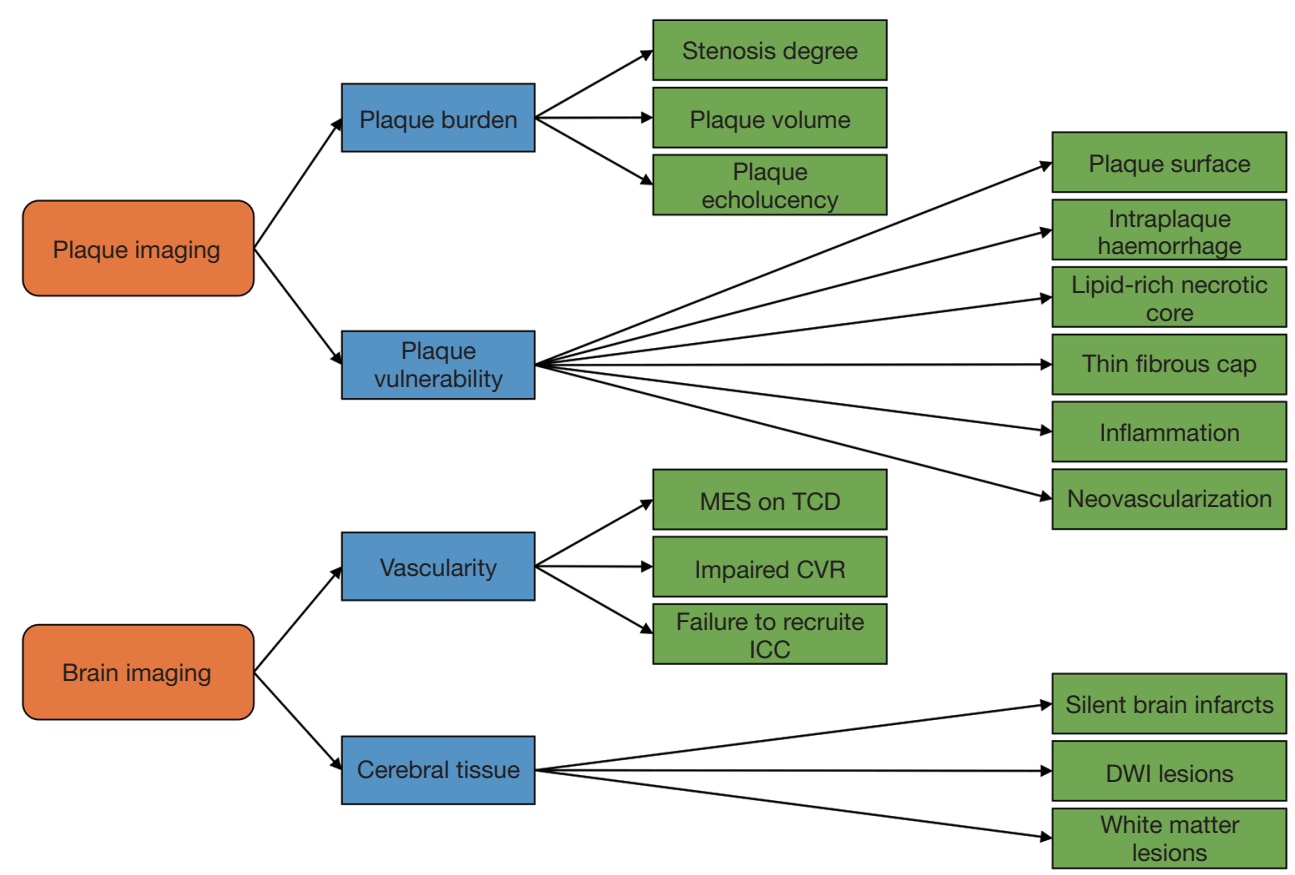

Figure 1 Imaging features of plaque and brain associated with future events. MES, microembolic signals; TCD, transcranial Doppler; CVR, cerebrovascular reserve; ICC, intracranial collaterals; DWI, diffusion weighted imaging.

(Figure 1) that have potential to predict future or recurrent cerebral events in patients with atherosclerotic carotid artery disease. In the future, these imaging biomarkers might be used for individual patient risk stratification to optimize treatment strategies. Additionally, we provide recommendations for future study designs to create widespread comparable results. This review is structured as followed: (I) the translation of ex vivo into in vivo examination, (II) results of potential plaque and brain imaging features (III) developments in imaging modalities and (IV) discussion and future perspectives.

We present the following article in accordance with the Narrative Review reporting checklist (available at https:// dx.doi.org/10.21037/atm-21-1166).

\section{Methods}

We conducted literature searches for English published RCTs, prospective and retrospective cohort studies, guidelines, and reviews reporting on plaque and brain imaging biomarkers up to December 2020 using PubMed, EMBASE and Scopus. The following terms were used in various combinations: 'carotid artery disease or stenosis', 'atherosclerosis', 'stroke prevention', 'carotid endarterectomy', 'imaging biomarker', 'risk stratification' 'personalized medicine', 'stenosis degree', 'plaque volume', 'plaque echolucency', 'plaque surface', 'intraplaque haemorrhage', 'lipid-rich necrotic core', 'fibrous cap', 'inflammation', 'neovascularization', 'microembolic signals', 'cerebrovascular reserve', 'intracranial collaterals', 'silent brain infarcts', 'diffusion weighted imaging lesions' and 'white matter lesions'. Studies were critically reviewed for the extraction of qualitative and quantitative data. Citations in included articles were checked (backward citation searching) and articles citing relevant publications were studied (forward citation searching) as potential source of information.

\section{Translation of ex vivo plaque characterization into in vivo examination}

Numerous studies have attempted to identify characteristics of vulnerable plaques based on histological analysis. The Athero-Express (AE) study, an ongoing vascular biobank, started in 2002 with the prospective collection of vascular specimens obtained during CEA, baseline characteristics and clinical follow-up up to 3 years. Results of the AE study showed that patients with clinical key risk predictors, including advanced age, male gender and symptomatic 
presentation with less delay between presentation of index symptoms and CEA, experienced most benefit from CEA (10), and had developed the highest prevalence of vulnerable plaque characteristics (containing large lipid core, high inflammatory infiltration, and decreased smooth muscle cell content) (17). Other post-hoc analysis illustrated that plaque composition varied by clinical presentation and type of index event, showing that patients with ocular ischemic events had comparable plaque characteristics to asymptomatic patients, and had relatively less vulnerable plaque characteristics compared to patients with recent stroke or transient ischemic attack (TIA) (18). In addition to clinical symptoms, high risk plaques were also associated with the presence of silent ischemic brain lesions on imaging which are increasingly proposed as surrogate marker for stroke (19). A recent retrospective AE study investigated the presence of silent brain lesions on magnetic resonance diffusion weighted imaging (MR-DWI) during the waiting period between index event and CEA. The analysis revealed that intraplaque haemorrhage (IPH) was independently associated with the presence of ipsilateral DWI lesions (20). However, since post-surgery patients following plaque removal are no longer subjected to its potential high risk of future stroke, these studies are unable to determine the exact prognostic value of high-risk plaque characteristics in CEA patients. The introduction of in vivo imaging allows us to identify which plaque and brain characteristics confer an elevated risk on future events. Integration of high-risk imaging features in prediction algorithms (including age, gender, and other risk factors) may help to allocate patients to optimal medical therapy or revascularization procedures for stroke prevention.

\section{'High risk' imaging features of the atherosclerotic carotid plaque}

\section{Progression of stenosis degree}

Progression in the degree of stenosis is assumed to be an indicator of plaque instability, but the optimal way of measurement is debated. The Asymptomatic Carotid Stenosis and Risk of Stroke (ACSRS) study, the largest natural history cohort with 1,115 asymptomatic patients with $50-99 \%$ stenosis, showed that based on duplex ultrasound imaging the 8-year cumulative ipsilateral ischemic stroke risk was $0 \%$ for regressive stenosis, $9 \%$ in unchanged stenosis and $16 \%$ in progressive stenosis (21). Although patients with progression of stenosis appeared to have double the risk of ipsilateral stroke, stenosis progression did not improve the multivariate model (22), developed within the same cohort of patients, on top of other clinical and imaging risk factors. Main limitations of the study were low incidence of stenosis progression (19.8\%) and low event incidence in the progression group (19 out of total 59 strokes). A substudy of the Asymptomatic Carotid Surgery Trial 1 (ACST-1) analyzing patients randomized to medical therapy found that stenosis progression of $\geq 2$ categories (categories were classified as $0 \%$ to $49 \%$, $50 \%$ to $69 \%, 70 \%$ to $89 \%, 90 \%$ to $99 \%$, and $100 \%$ ) was an independent predictor for ipsilateral events, including TIA, amaurosis fugax, stroke and symptomatic carotid occlusion (23). However, progression of two categories was demonstrated in only 50 out of total 1,469 (3.4\%) patients. Plaque progression does not seem very common, but when plaques tend to progress, they may reflect a high risk profile.

\section{Progression of plaque volume}

Progression in total plaque volume may be a more promising predictor for stroke because of its 3-dimensional (3D) disposition. In 306 patients attending vascular prevention clinics, 3D US determined progression of total plaque volume remained a significant predictor of cardiovascular events after adjustment for coronary risk factors (24). In a smaller prospective longitudinal study, 63 symptomatic patients with ipsilateral 30-69\% carotid stenosis underwent ultrasound and first and second MRI at baseline and $\geq 6$ months after the first scan with volume measurement of the carotid plaque (25). The annual progression of carotid wall volume was significantly associated with ipsilateral recurrent events adjusted for confounding factors, suggesting that volume progression might be used in a risk model for low degree stenosis patients. New advanced computational models are developed to reconstruct carotid anatomy and to monitor development of the plaque over time (26). However, the clinical relevance of these computational models to predict future stroke risk needs to be determined.

\section{Plaque echolucency}

Duplex ultrasound (DUS) derived echolucent plaques have been shown to correspond to high content of LRNC and IPH, while echogenic plaques correspond to high fibrous content (27). A meta-analysis including 7,557 asymptomatic patients found a significant association between echolucent 
plaques and risk on future ipsilateral stroke in patients with 0-99\% stenosis [relative risk (RR) 2.31] and even higher in patients with $\geq 50 \%$ stenosis (RR 2.61) (28). This was verified in the 5 -year post-hoc analysis of the medical randomized arm in the ACST-1 (29) and a more recent meta-analysis (30) of both symptomatic and asymptomatic patients. Currently, the juxta-luminal black area (JBA), representing the echolucent areas of both LRNC and IPH close to the lumen without a visible $\mathrm{FC}$, has been proposed to be a valuable variation of echolucency (31). The ACSRS cohort showed in multivariate analysis that JBA was still a significant independent predictor for ipsilateral ischemic events after correction for other at risk plaque features (32). Based on this study (I) $\geq 8 \mathrm{~mm}^{2}$ was suggested as a relevant cut-off value, while (II) JBA having a linear correlation with future stroke, so might be used as feature for the stratification of a high-risk on future stroke cohort. However, in this study BMT strategies where outdated compared to the current modern standards, making the reported results less reliable. Currently, the optimal way to differentiate echolucent from echogenic plaques is still under debate. Future studies are needed to provide standardized definitions of echolucency with clear thresholds, assessed by standardized methods to create outcomes that can be incorporated in multifactorial risk stratification models.

\section{Plaque surface}

A focal depression, causing an irregular surface, suggests plaque ulceration which could induce plaque rupturing, causing thrombosis and embolization. Pooled data from the North American Symptomatic Carotid Endarterectomy Trial (NASCET) and ECST-1 showed that medically treated patients presenting with irregular or ulcerated plaques on intra-arterial angiography had a significant higher risk on recurrent ipsilateral stroke at 2 years compared to patients without ulceration (33). The presence of plaque irregularity can be visualized using US, CT, and MRI, whereas CT with contrast agent is superior because of best spatial resolution. US is suboptimal, but its accuracy can be improved by contrast enhanced microbubbles (14). The exact value of surface morphology remains unclear since ulceration could also be an expression of previous rupture concluding that future studies need to verify its value (34).

\section{Plaque composition}

MRI has been suggested as the most suitable imaging technique for the assessment of IPH, LRNC and FC $(14,35)$.

Intraplaque haemorrhage is thought to be caused by leakage of intraplaque neovessels and fissuring of the plaque causing formation of the intraluminal thrombus into the plaque (36). Today, IPH is seen as the strongest predictor for ischemic stroke and guidelines already suggest to use this feature to select high-risk asymptomatic patients for whom intervention is contemplated $(5,37)$. However, a large proportion of patients with presence of IPH will not experience future or recurrent ischemic cerebral events. It would be valuable for future studies to visualize the different stages of IPH $(38,39)$ to potentially optimize the specificity of this imaging feature, or to monitor the effect of plaque stabilizing medication in sequential imaging reports.

Lipid-rich necrotic core arises from differentiation of monocytes into lipoprotein filled foam cells that precipitate in the vessel wall, and is later covered by the FC. The thrombogenic necrotic core is exposed to the luminal blood when the FC fissures or ruptures, which can activate atherothrombosis (40). In a prospective MRI study with 214 atherosclerotic disease participants, the amount of lipid plaque content was significantly associated with cardiovascular events (41).

Fibrous cap is a layer of collagen tissue that is thought to be prone to be disrupted by decreased smooth muscle cell content or degraded by matrix metalloproteinases excreted by macrophages, resulting from inflammatory and hemodynamic factors (42). A thick and intact FC is believed to be associated with low-risk plaque rupture, whereas a thin or fissured plaque is associated with high risk plaque rupture (43). Accurate visualizing of the FC, however, requires MR imaging with gadolinium contrast agent (44).

A meta-analysis showed that IPH, a thin or ruptured FC and LRNC were associated with increased risk of future ipsilateral stroke or TIA in asymptomatic [IPH with hazard ratio (HR) 3.66 and LRNC HR 5.74], and in symptomatic patients (IPH with HR 5.86, LRNC HR 2.41 and FC HR 6.30) who both received BMT without surgical or endovascular intervention (45). However, limitations included heterogeneity in patient selection, imaging strategy and outcome. Further, the analysis was based on nonrandomized studies only, potentially introducing selection bias since medically treated patients may differ from surgical patients in plaque morphology and cardiovascular risk profile. Future studies need to verify if quantitative measurement of these imaging features, and in case of IPH 
also staging, might improve their ability to predict future events.

\section{Plaque activity}

Plaque inflammation and intraplaque neovascularization might give valuable insights in the fundamental processes of plaque activity and progression in atherosclerosis but both are challenging to objectify on imaging $(46,47)$.

Inflammation is activated when cholesterol-filled lowdensity lipoproteins cross the endothelium and become oxidized, resulting in the attraction of monocytes to the lesion. Inflammatory cell infiltration is thought to be maintained during all phases of the atherosclerotic process. Studies found that plaque inflammation can be noninvasively visualized on positron emission tomography (PET)/CT by increased ${ }^{18} \mathrm{~F}$-fluorodeoxyglucose $\left({ }^{18} \mathrm{~F}-\mathrm{FDG}\right)$ uptake, and inflammation is more prevalent in the carotid arteries ipsilateral to the recent ischemic event (48). A recent study constructed a risk score (SCAIL [Symptomatic Carotid Atheroma Inflammation Lumen-Stenosis]) based on plaque ${ }^{18} \mathrm{~F}$-FDG uptake and lumen stenosis, that improved the prediction of early ipsilateral recurrent stroke after correction for established risk factors, even in patients with mild to moderate stenosis (49). FDG PET/CT seemed promising for the detection of inflammation but enables with poor spatial resolution, which impedes simultaneous tracing of other characteristics.

Neovascularization arises from hypoxic areas and inflammatory stimuli in the plaque, resulting in immature neovessels that are thought to be prone to rupture or leak (50). The extension of neovascularity, measured as degree of adventitial enhancement on dynamic contrast enhancement MRI (DCE-MRI), was independently associated with previous ischemic events after controlling for age, sex, cardiovascular risk factors, wall thickness, and stenosis (51). However, because of the small size and motion artifacts of the vessel wall the value of DCEMRI might be limited for stratification in clinical setting. A recent meta-analysis postulated that quantitative measurement using contrast enhanced US (CEUS) can be a promising alternative for the detection of intraplaque neovascularization, but its predictive value has to be verified first in prospective clinical studies (52).

Currently, imaging of plaque inflammation and neovascularization is expensive and time consuming and should be researched more thoroughly to derive predictors with clear thresholds that can be used in risk stratification analysis.

\section{'High risk' imaging features of the brain}

\section{Spontaneous embolization on TCD}

Spontaneous microembolic signals (MES) on transcranial Doppler (TCD) ultrasonography might arise from unstable plaques of the carotid artery and/or other proximal sources or from impaired washout of emboli because of poor hemodynamic compensation. A study of 319 asymptomatic carotid patients reported that the presence of $>2$ microemboli during 1 hour of TCD monitoring increased the risk of ipsilateral ischemic stroke compared to patients without MES at one year follow-up ( $15.6 \%$ vs. $1.0 \%$, respectively; $\mathrm{P}<0.0001$ ) (53). Five years later, the same group showed that with the implementation of more intensive BMT there was a significant reduction in both MES on TCD and cardiovascular events. Despite this, microemboli detection remained a reliable parameter to differentiate patients at a higher risk of the composite endpoint (including stroke, death, myocardial infarction, or CEA) (54). The Asymptomatic Carotid Emboli Study (ACES) confirmed these findings in a prospective international multicenter study in 467 patients (55). They showed an over 5 -fold increase in risk of ipsilateral stroke in patients with $\geq 1$ MES on TCD compared with those without MES at 2 years. A subgroup of the ACES study had ultrasound images available for visual echolucent plaque assessment. The combination of MES with plaque echolucency offered the highest predictive value in the risk of ipsilateral stroke, even after correction for risk factors, stenosis severity and antiplatelet therapy (56).

The clinical relevance of MES on TCD in the waiting period between the index event and intervention is a debated subject. In a study of 200 symptomatic patients with $>50 \%$ stenosis, patients were followed prospectively until stroke, revascularization of the carotid, death, or study completion at 90 days after recruitment occurred (57). After correction of age, sex, smoking, hypertension, time from last symptoms, and degree of stenosis, presence of $\geq 1$ MES during 1 hour of monitoring remained a significant predictor for short-term ipsilateral stroke. Additionally, another study showed that symptomatic patients treated within the early time frame showed significant higher MES prevalence than $>14$ days after index event, but did not reach a significant increased risk of recurrent cerebral ischemic events prior to the expedited CEA (58). However, limitations were a small study cohort and the possibility of missed MES because of the relatively short TCD monitoring of 30 minutes. TCD measured MES rate might 
help to optimize risk stratification, but the optimal TCD recording time and amount of MES needs to be verified first.

\section{Impaired cerebrovascular reserve (CVR)}

Ischemic stroke is mainly caused by embolisms but can also be generated by hemodynamic compromise resulting from hypoperfused regions of the brain. The reserve capacity of autoregulation of the brain, also known as CVR, can be tested by flow measurements before and after vasodilator stimuli, often done by TCD ultrasonography carbon dioxide $\left(\mathrm{CO}_{2}\right)$ reactivity (59). A meta-analysis of both symptomatic and asymptomatic patients with carotid stenosis showed significant association of impaired CVR with future development of stroke (OR 3.86) based on TCD or nuclear medicine techniques (60). Limitations of this study included variability in CVR measurement, heterogeneous inclusion of study participants (definition of asymptomatic versus asymptomatic varied) and variably in study endpoints. However, the most recent meta-analysis including exclusively TCD $\mathrm{CO}_{2}$ reactivity in severe stenosis patients $(\geq 70 \%)$ not scheduled for intervention supported these findings, showing an independent association of impaired CVR with ipsilateral ischemic stroke (61). Implementation of CVD status might be contributive to identify patients at higher risk of stroke, but the most optimal way of measurement first needs to be verified before using this feature in major clinical trials.

\section{Failure to recruit intracranial collaterals}

Blood supply through cerebral collateral circulation may improve regional cerebral hemodynamics and may protect against ischemic events (62). NASCET data showed that the presence of intracranial collateral supply based on conventional angiography after carotid or vertebral injection increased with the severity of stenosis (63). Also, absence of collaterals was an independent predictor for ipsilateral stroke ( $27.8 \%$ vs. $11.3 \%)$ and TIA (36.1\% vs. $19.1 \%)$ in medically treated patients with severe stenosis compared to patients with presence of collaterals. Last, the 2-year absolute risk reduction on ipsilateral stroke effectuate by CEA versus BMT was $5.4 \%$ when having collateral supply and $19.4 \%$ without. The absence of intracranial collaterals might help to identify a subgroup with higher risk on future stroke. The imaging modality in the NASCET study to visualize recruitment of intracranial collaterals is no longer used in today's clinical practice, however advanced CT/MRI imaging techniques using contrast angiography might serve as good alternative for measurement of collateral capacity (64).

\section{Silent brain infarcts}

Silent brain infarcts (SBI) are cerebral infarcts without clinical symptoms which can be detected on MRI [T2 or fluid-attenuated inversion recovery (FLAIR)] with higher sensitivity than on CT and are associated with increased risk of stroke in the general population (65).

The ACSRS study reported a subgroup analysis of 462 asymptomatic $60-99 \%$ stenosis patients who underwent brain CT imaging at baseline for SBI scoring (66). Results showed that at 8-year follow-up patients with SBIs in the ipsilateral hemisphere had a significant higher cumulative risk on ipsilateral stroke compared to patients without SBIs (28.8\% vs. 8.0\%; HR 3.0). In 104 asymptomatic patients with $\geq 50 \%$ stenosis without a history of stroke/ TIA, a higher prevalence of cortical SBIs ipsilateral to the index carotid artery was seen compared to the contralateral side $(14.7 \%$ vs. $3.9 \% ; \mathrm{P}=0.0045)$, whereas the presence of lacunar SBIs was not significantly different (67). This is consistent with the idea that cortical infarction is more likely to be caused by emboli from a proximal source. This study demonstrated that asymptomatic atherosclerotic carotid disease is associated with higher presence of ipsilateral SBIs and the presence of ipsilateral SBIs might be useful for risk stratification.

The ESVS guidelines suggest to use the presence of SBIs for selecting asymptomatic patients at higher-risk for stroke for revascularization. This recommendation is based on a study using CT imaging for SBI detection, so results might indicate an underestimation of the true incident rate of SBIs. Future prospective observational studies are needed to validate the current recommendations and to obtain consensus in imaging techniques and diagnostic criteria of SBIs.

\section{Diffusion weighted imaging (DWI)}

DWI lesions represent acute ischemic brain areas that can be detected on brain imaging within minutes after a hypoxic event and remain visible for several days (19). The MRI substudy within International Carotid Stenting Study (ICSS) demonstrated that patients in the CAS group with DWI positive scans had a significantly higher risk on any recurrent stroke or TIA than patients with DWI 
negative scans (68). This association was not seen in the CEA group, however only $16.8 \%$ of CEA patients had new DWI lesions on post-procedural MRI, compared to $50 \%$ in the CAS group. A 2019 meta-analysis including 6,970 revascularisation procedures showed a strong association between DWI positive patients and procedural stroke and calculated that a $90 \%$ sample size reduction could be achieved when using DWI-lesions instead of the classic clinical endpoint to detect an underlying procedural stroke risk difference of $3 \%$ (69). There was excessive heterogeneity in the included studies, implying the need for future studies to verify the optimal timing of the postprocedural scan and create harmonized scanning protocols.

\section{White matter lesions (WML)}

WML are visible on brain CT and MR imaging and are closely associated with first and recurrent stroke, cognitive impairment and cerebrovascular death (70). Although the strong association with cerebrovascular risk, the pathogenesis is still not well understood and is likely to be multifactorial (71).

In 2002, the NASCET showed that CEA treated patients with widespread WMLs had a significant higher perioperative 30 day risk on stroke or death compared to those without (13.9\% vs. $5.3 \%)$ (72). This association was also seen on the long-term, in both surgical and medically treated groups. On the contrary, a recent study based on 1,038 patients from the AE biobank observed that presence of WMLs and lacunar infarcts are associated with 3-year risk of cardiovascular death in symptomatic patients undergoing CEA, but were not predictive for perioperative or long-term risk of stroke (73). These results are consistent with earlier finding in the ICSS study, where no association between WMLs and perioperative stroke risk in CEA patients was confirmed (74). A possible explanation for the discrepancy is the improvement of BMT and surgical techniques in the more recent studies. WMLs volume might be a diagnostic and prognostic predictor for overall cerebrovascular disease with potential for medical therapy implications, however the clinical relevance of WMLs for the prediction of stroke remains controversial.

\section{Development in imaging modalities}

Assessment of the carotid plaque was for long time solely dependent on non-invasive B-mode US alone or in combination with color Doppler flow since its wide availability, low cost and low risk. However, this type of imaging is subjected to the operator's ability and interand intra-observer variability. Advances in high-resolution MRI and CT imaging have enabled higher accuracy in the differentiation of plaque burden and vulnerability (14). New developments in ultrasound, including contrast-enhanced ultrasound (CEUS), 3D ultrasound and intravenous US (IVUS), may provide more accurate and reliable characterization of the plaque, but their clinical value has to be research first in larger prospective studies (75). At all times, ultrasound based techniques remain operator dependent thereby limiting generalizability of findings. Furthermore, nuclear medicine and molecular imaging techniques have gained more attention. These imaging modalities can visualize biologic insight at molecular and cellular level and may distinguish vulnerable from nonvulnerable carotid plaques (76). Contemporary results are based on small investigational studies, so future larger prospective longitudinal studies are needed to confirm the clinical potential of these imaging strategies. In the future, these imaging modalities might also be used as in vivo validation instead of ex vivo histological validation for some plaque characteristics. In addition, several randomized placebo controlled trials, such as the CANTOS (77), COLCOT (78) and LoDoCo2 trial (79), have demonstrated that anti-inflammatory therapy lowered the risk on recurrent cardiovascular events in patients with coronary disease. Plaque inflammation on molecular imaging may have the potential to visualize the effect of anti-inflammatory therapy on the culprit plaque in atherosclerotic carotid artery disease. Last, these imaging modalities might not only have diagnostic utility, but also therapeutic applications for cardiovascular medicine. In the brain imaging field, using 7Tesla MRI have led to new methods to measure the structure and function of cerebral perforators and might evaluate the effect of revascularization procedure on cerebral arterial function (80).

New developments of carotid imaging, such as CEUS and ${ }^{18} \mathrm{~F}$-FDG uptake on PET/CT, can give us more insights in the pathophysiological course of atherosclerotic disease, but their capability to improve treatment strategies is not yet researched thoroughly enough for today's clinical practice.

\section{Discussion and future perspectives}

A large number of studies have suggested specific imaging features to be associated with increased risk of stroke. The main findings of imaging biomarkers discussed in this review are summarized in Table 1. However, their results 
Table 1 Overview of imaing features associated with an increased risk of ischemic cerebral events in patients with atherosclerotic carotid artery disease

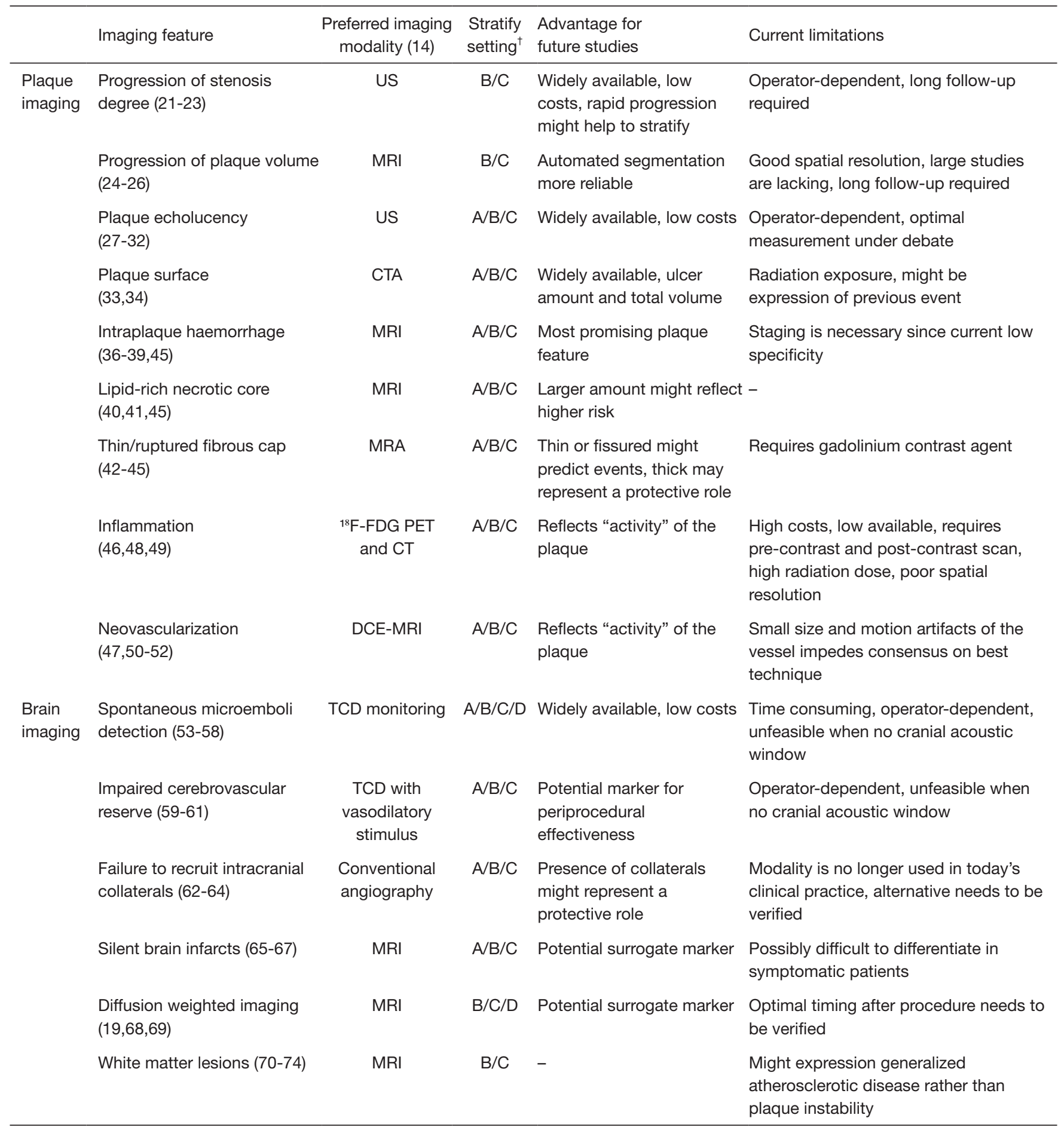

\footnotetext{
${ }^{\dagger}, \mathrm{A}$ : applicable for acute decision-making (CEA/CAS versus BMT); B: applicable for follow-up; C: marker medical add-on therapy, D: marker periprocedural risk. US, ultrasound; MRI, magnetic resonance imaging; MRA, magnetic imaging angiography; ${ }^{18} \mathrm{~F}-\mathrm{FDG} \mathrm{PET}$, 18F-fluorodeoxyglucose positron emission tomography; CTA, computed tomography angiography; CT, computed tomography; DCE-MRI, dynamic contrast enhanced-MRI; TCD, transcranial Doppler.
} 


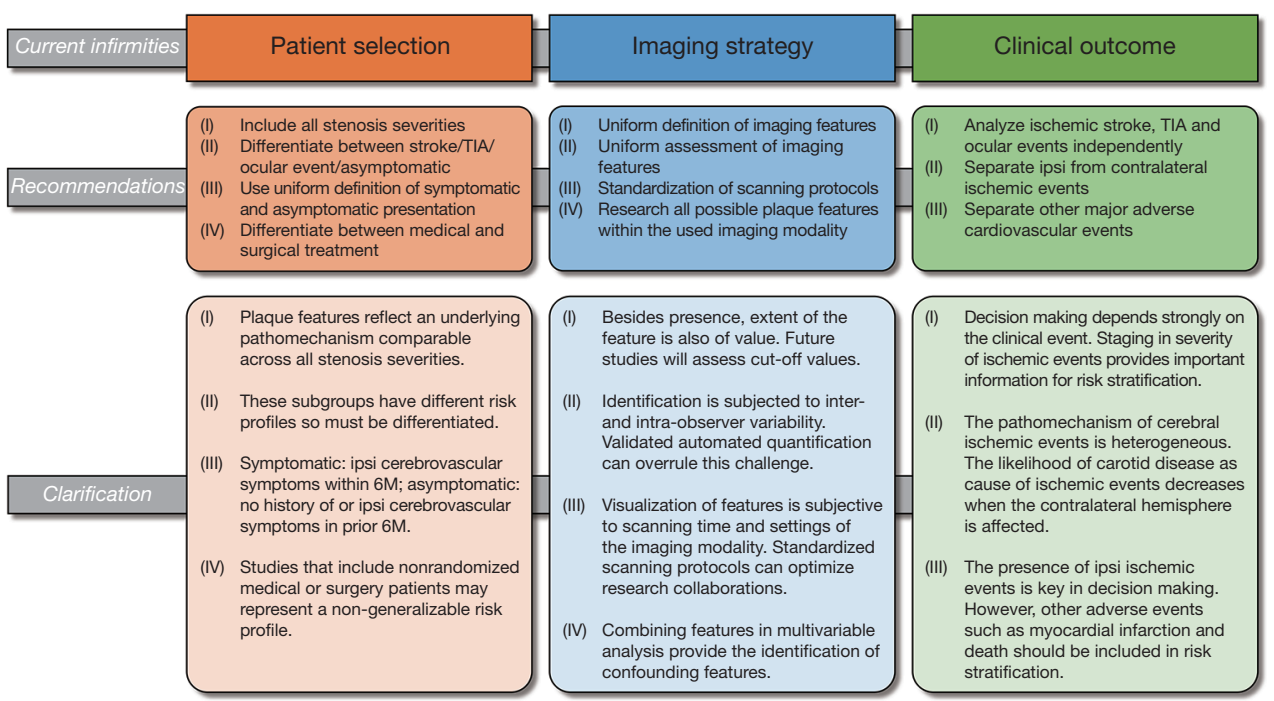

Figure 2 Optimization of the designs in future studies is highly necessary to establish comparable results. A flowchart was constructed to provide recommendations for future studies. TIA, transient ischemic attack; $M$, months.

need further validation and studies lack sufficient power to incorporate the specific parameters in risk stratification for today's clinical practice. First, the features have overall been studied in small populations or substudies and the incidence of the clinical end-point ipsilateral stroke occurs at low rates. Second, there is enormous heterogeneity within patients selection including different definitions of symptomatic and asymptomatic patients and stenosis grade; within the definition and criteria for the imaging modalities resulting in a lack of consistent determinants; and within clinical end-points including inconsistent reports of contra- and ipsilateral events and aggregation of other cerebrovascular events (i.e., myocardial infarction and death). Third, BMT strategies evolved overtime resulting in the inability to compare old with current studies and therefore the inability to draw conclusions from older studies for present treatment strategies. Last, a multicenter study that incorporated all imaging features is still lacking, leaving the mutual association of characteristics unknown. Concluding, optimization of the designs in future studies is highly necessary to establish comparable results. Based on the shortcomings of the current literature, we have defined recommendations for future studies (Figure 2).

Currently, the TAXINOMISIS Project, a prospective observational multicenter clinical trial, aims to develop a risk stratification model for asymptomatic carotid disease patients with $50-99 \%$ stenosis on the risk of cerebrovascular events based on clinical, circulating and imaging biomarkers (81).
Such studies and future RCTs with patients under current optimal medical treatment are warranted to identify the potential value of plaque and brain imaging features for the risk stratification in both symptomatic and asymptomatic carotid disease patients. Risk algorithms that can predict future stroke are already demonstrated (22), and new promising diagnostic imaging tools should be incorporated for optimization. However, it should be noted that clinical risk factors form the basis of stroke prediction models, since they are easily obtainable and less costly than imaging and circulating biomarkers. Therefore, future studies first need to verify the synergistic prognostic ability of imaging biomarker on top of the clinical biomarkers in prediction models that estimate the risk of future or recurrent stroke before implementation in clinical practice.

Besides the high risk of thromboembolic ischemic cerebral events, patients with carotid artery disease are also at risk for compromised cerebral haemodynamics, possibly causing cognitive decline and cerebral ischemia due to chronic cerebral hypoperfusion $(82,83)$. A recent study showed the ability of revascularization procedures to normalize characteristic alternations of brain perfusion present in the territory of the affected carotid artery at baseline, however this study was not able to observe improvements in cognitive performance (84). This shows the importance for future research on the insights of cerebral haemodynamic impairment as surrogate marker for ischemic stroke and of cognition decline as potential 
additional outcome indicator to improve treatment strategy in carotid artery disease.

Creating a validated risk algorithm with integration of all relevant biomarkers seems a reasonable way to overcome the current challenge of over- and undertreatment and approaches the ultimate goal of performing personalized evidence-based treatment decision making in carotid atherosclerotic disease. Currently, a single imaging modality that can reliably identify all imaging features at risk for future stroke is still lacking. However, in the meantime, the presence of one or more imaging features discussed in this review might already support the selection of high risk for stroke patients for revascularization.

\section{Acknowledgments}

Funding: None.

\section{Footnote}

Reporting Checklist: The authors have completed the Narrative Review reporting checklist. Available at https:// dx.doi.org/10.21037/atm-21-1166

Conflicts of Interest: All authors have completed the ICMJE uniform disclosure form (available at https://dx.doi. org/10.21037/atm-21-1166). The authors have no conflicts of interest to declare.

Ethical Statement: The authors are accountable for all aspects of the work in ensuring that questions related to the accuracy or integrity of any part of the work are appropriately investigated and resolved.

Open Access Statement: This is an Open Access article distributed in accordance with the Creative Commons Attribution-NonCommercial-NoDerivs 4.0 International License (CC BY-NC-ND 4.0), which permits the noncommercial replication and distribution of the article with the strict proviso that no changes or edits are made and the original work is properly cited (including links to both the formal publication through the relevant DOI and the license). See: https://creativecommons.org/licenses/by-nc-nd/4.0/.

\section{References}

1. Krishnamurthi RV, Ikeda T, Feigin VL, et al. Global, Regional and Country-Specific Burden of Ischaemic
Stroke, Intracerebral Haemorrhage and Subarachnoid Haemorrhage: A Systematic Analysis of the Global Burden of Disease Study 2017. Neuroepidemiology 2020;54:171-9.

2. North American Symptomatic Carotid Endarterectomy Trial Collaborators; Barnett HJM, Taylor DW, et al. Beneficial effect of carotid endarterectomy in symptomatic patients with high-grade carotid stenosis. N Engl J Med 1991;325:445-53.

3. Randomised trial of endarterectomy for recently symptomatic carotid stenosis: final results of the MRC European Carotid Surgery Trial (ECST) Lancet 1998;351:1379-87.

4. Endarterectomy for asymptomatic carotid artery stenosis. Executive Committee for the Asymptomatic Carotid Atherosclerosis Study. JAMA 1995;273:1421-8.

5. Naylor AR, Ricco JB, de Borst GJ, et al. Editor's Choice - Management of Atherosclerotic Carotid and Vertebral Artery Disease: 2017 Clinical Practice Guidelines of the European Society for Vascular Surgery (ESVS). Eur J Vasc Endovasc Surg 2018;55:3-81.

6. Kernan WN, Ovbiagele B, Black HR, et al. Guidelines for the prevention of stroke in patients with stroke and transient ischemic attack: a guideline for healthcare professionals from the American Heart Association/ American Stroke Association. Stroke 2014;45:2160-236.

7. Naylor AR, Sillesen H, Schroeder TV, et al. Clinical and imaging features associated with an increased risk of early and late stroke in patients with symptomatic carotid disease. Eur J Vasc Endovasc Surg 2015;49:513-23.

8. Naylor AR. Why is the management of asymptomatic carotid disease so controversial? Surgeon 2015;13:34-43.

9. Keyhani S, Cheng EM, Hoggatt KJ, et al. Comparative Effectiveness of Carotid Endarterectomy vs Initial Medical Therapy in Patients With Asymptomatic Carotid Stenosis. JAMA Neurol 2020;77:1110-21.

10. Rothwell PM, Eliasziw M, Gutnikov SA, et al. Endarterectomy for symptomatic carotid stenosis in relation to clinical subgroups and timing of surgery. Lancet 2004;363:915-24.

11. Karlsson L, Kangefjärd E, Hermansson S, et al. Risk of Recurrent Stroke in Patients with Symptomatic Mild (20-49\% NASCET) Carotid Artery Stenosis. Eur J Vasc Endovasc Surg 2016;52:287-94.

12. Davies MJ. Stability and instability: two faces of coronary atherosclerosis. The Paul Dudley White Lecture 1995. 
Circulation 1996;94:2013-20.

13. Howard DP, van Lammeren GW, Rothwell PM, et al. Symptomatic carotid atherosclerotic disease: correlations between plaque composition and ipsilateral stroke risk. Stroke 2015;46:182-9.

14. Saba L, Saam T, Jäger HR, et al. Imaging biomarkers of vulnerable carotid plaques for stroke risk prediction and their potential clinical implications. Lancet Neurol 2019;18:559-72.

15. Kamtchum-Tatuene J, Noubiap JJ, Wilman AH, et al. Prevalence of High-risk Plaques and Risk of Stroke in Patients With Asymptomatic Carotid Stenosis: A Metaanalysis. JAMA Neurol 2020;77:1524-35.

16. Hyafil F, Schindler A, Sepp D, et al. High-risk plaque features can be detected in non-stenotic carotid plaques of patients with ischaemic stroke classified as cryptogenic using combined (18)F-FDG PET/MR imaging. Eur J Nucl Med Mol Imaging 2016;43:270-9.

17. Hellings WE, Moll FL, de Kleijn DP, et al. 10-years experience with the Athero-Express study. Cardiovasc Diagn Ther 2012;2:63-73.

18. Howard DP, van Lammeren GW, Redgrave JN, et al. Histological features of carotid plaque in patients with ocular ischemia versus cerebral events. Stroke 2013;44:734-9.

19. Rots ML, van der Lugt A, de Borst GJ, et al. Surrogate Markers and Reporting Standards for Outcome After Carotid Intervention. Eur J Vasc Endovasc Surg 2019;58:794-5.

20. Rots ML, Timmerman N, de Kleijn DPV, et al. Magnetic Resonance Imaging Identified Brain Ischaemia in Symptomatic Patients Undergoing Carotid Endarterectomy Is Related to Histologically Apparent Intraplaque Haemorrhage. Eur J Vasc Endovasc Surg 2019;58:796-804.

21. Kakkos SK, Nicolaides AN, Charalambous I, et al. Predictors and clinical significance of progression or regression of asymptomatic carotid stenosis. J Vasc Surg 2014;59:956-967.e1.

22. Nicolaides AN, Kakkos SK, Kyriacou E, et al. Asymptomatic internal carotid artery stenosis and cerebrovascular risk stratification. J Vasc Surg 2010;52:1486-1496.e1-5.

23. Hirt LS. Progression rate and ipsilateral neurological events in asymptomatic carotid stenosis. Stroke 2014;45:702-6.

24. Wannarong T, Parraga G, Buchanan D, et al. Progression of carotid plaque volume predicts cardiovascular events.
Stroke 2013;44:1859-65.

25. Lu M, Peng P, Cui Y, et al. Association of Progression of Carotid Artery Wall Volume and Recurrent Transient Ischemic Attack or Stroke: A Magnetic Resonance Imaging Study. Stroke 2018;49:614-20.

26. Sakellarios AI, Stefanou K, Siogkas P, et al. Novel methodology for 3D reconstruction of carotid arteries and plaque characterization based upon magnetic resonance imaging carotid angiography data. Magn Reson Imaging 2012;30:1068-82.

27. El-Barghouty NM, Levine T, Ladva S, et al. Histological verification of computerised carotid plaque characterisation. Eur J Vasc Endovasc Surg 1996;11:414-6.

28. Gupta A, Kesavabhotla K, Baradaran H, et al. Plaque echolucency and stroke risk in asymptomatic carotid stenosis: a systematic review and meta-analysis. Stroke 2015;46:91-7.

29. Huibers A, de Borst GJ, Bulbulia R, et al. Plaque Echolucency and the Risk of Ischaemic Stroke in Patients with Asymptomatic Carotid Stenosis Within the First Asymptomatic Carotid Surgery Trial (ACST-1). Eur J Vasc Endovasc Surg 2016;51:616-21.

30. Jashari F, Ibrahimi P, Bajraktari G, et al. Carotid plaque echogenicity predicts cerebrovascular symptoms: a systematic review and meta-analysis. Eur J Neurol 2016;23:1241-7.

31. Griffin MB, Kyriacou E, Pattichis C, et al. Juxtaluminal hypoechoic area in ultrasonic images of carotid plaques and hemispheric symptoms. J Vasc Surg 2010;52:69-76.

32. Kakkos SK, Griffin MB, Nicolaides AN, et al. The size of juxtaluminal hypoechoic area in ultrasound images of asymptomatic carotid plaques predicts the occurrence of stroke. J Vasc Surg 2013;57:609-618.e1; discussion 617-8.

33. Naylor AR, Rothwell PM, Bell PR, et al. Overview of the principal results and secondary analyses from the European and North American randomised trials of endarterectomy for symptomatic carotid stenosis. Eur J Vasc Endovasc Surg 2003;26:115-29.

34. van Gils MJ, Homburg PJ, Rozie S, et al. Evolution of atherosclerotic carotid plaque morphology: do ulcerated plaques heal? A serial multidetector CT angiography study. Cerebrovasc Dis 2011;31:263-70.

35. den Hartog AG, Bovens SM, Koning W, et al. Current status of clinical magnetic resonance imaging for plaque characterisation in patients with carotid artery stenosis. Eur J Vasc Endovasc Surg 2013;45:7-21.

36. Michel JB, Virmani R, Arbustini E, et al. Intraplaque 
haemorrhages as the trigger of plaque vulnerability. Eur Heart J 2011;32:1977-85, 1985a, 1985b, 1985c.

37. Schindler A, Schinner R, Altaf N, et al. Prediction of Stroke Risk by Detection of Hemorrhage in Carotid Plaques: Meta-Analysis of Individual Patient Data. JACC Cardiovasc Imaging 2020;13:395-406.

38. Derksen WJ, Peeters $W$, van Lammeren GW, et al. Different stages of intraplaque hemorrhage are associated with different plaque phenotypes: a large histopathological study in 794 carotid and 276 femoral endarterectomy specimens. Atherosclerosis 2011;218:369-77.

39. Mura M, Della Schiava N, Long A, et al. Carotid intraplaque haemorrhage: pathogenesis, histological classification, imaging methods and clinical value. Ann Transl Med 2020;8:1273.

40. Bentzon JF, Otsuka F, Virmani R, et al. Mechanisms of plaque formation and rupture. Circ Res 2014;114:1852-66.

41. Sun J, Zhao XQ, Balu N, et al. Carotid Plaque Lipid Content and Fibrous Cap Status Predict Systemic CV Outcomes: The MRI Substudy in AIM-HIGH. JACC Cardiovasc Imaging 2017;10:241-9.

42. Galis ZS, Sukhova GK, Lark MW, et al. Increased expression of matrix metalloproteinases and matrix degrading activity in vulnerable regions of human atherosclerotic plaques. J Clin Invest 1994;94:2493-503.

43. Demarco JK, Ota H, Underhill HR, et al. MR carotid plaque imaging and contrast-enhanced MR angiography identifies lesions associated with recent ipsilateral thromboembolic symptoms: an in vivo study at 3T. AJNR Am J Neuroradiol 2010;31:1395-402.

44. Cai J, Hatsukami TS, Ferguson MS, et al. In vivo quantitative measurement of intact fibrous cap and lipidrich necrotic core size in atherosclerotic carotid plaque: comparison of high-resolution, contrast-enhanced magnetic resonance imaging and histology. Circulation 2005;112:3437-44.

45. Gupta A, Baradaran H, Schweitzer AD, et al. Carotid plaque MRI and stroke risk: a systematic review and metaanalysis. Stroke 2013;44:3071-7.

46. Truijman MT, Kwee RM, van Hoof RH, et al. Combined 18F-FDG PET-CT and DCE-MRI to assess inflammation and microvascularization in atherosclerotic plaques. Stroke 2013;44:3568-70.

47. Horie N, Morofuji Y, Morikawa M, et al. Communication of inwardly projecting neovessels with the lumen contributes to symptomatic intraplaque hemorrhage in carotid artery stenosis. J Neurosurg 2015;123:1125-32.

48. Chaker S, Al-Dasuqi K, Baradaran H, et al. Carotid Plaque
Positron Emission Tomography Imaging and Cerebral Ischemic Disease. Stroke 2019;50:2072-9.

49. Kelly PJ, Camps-Renom P, Giannotti N, et al. A Risk Score Including Carotid Plaque Inflammation and Stenosis Severity Improves Identification of Recurrent Stroke. Stroke 2020;51:838-45.

50. Sluimer JC, Gasc JM, van Wanroij JL, et al. Hypoxia, hypoxia-inducible transcription factor, and macrophages in human atherosclerotic plaques are correlated with intraplaque angiogenesis. J Am Coll Cardiol 2008;51:1258-65.

51. Qiao Y, Etesami M, Astor BC, et al. Carotid plaque neovascularization and hemorrhage detected by MR imaging are associated with recent cerebrovascular ischemic events. AJNR Am J Neuroradiol 2012;33:755-60.

52. Schinkel AFL, Bosch JG, Staub D, et al. ContrastEnhanced Ultrasound to Assess Carotid Intraplaque Neovascularization. Ultrasound Med Biol 2020;46:466-78.

53. Spence JD, Tamayo A, Lownie SP, et al. Absence of microemboli on transcranial Doppler identifies lowrisk patients with asymptomatic carotid stenosis. Stroke 2005;36:2373-8.

54. Spence JD, Coates V, Li H, et al. Effects of intensive medical therapy on microemboli and cardiovascular risk in asymptomatic carotid stenosis. Arch Neurol 2010;67:180-6.

55. Markus HS, King A, Shipley M, et al. Asymptomatic embolisation for prediction of stroke in the Asymptomatic Carotid Emboli Study (ACES): a prospective observational study. Lancet Neurol 2010;9:663-71.

56. Topakian R, King A, Kwon SU, et al. Ultrasonic plaque echolucency and emboli signals predict stroke in asymptomatic carotid stenosis. Neurology 2011;77:751-8.

57. Markus HS, MacKinnon A, et al. Asymptomatic embolization detected by Doppler ultrasound predicts stroke risk in symptomatic carotid artery stenosis. Stroke 2005;36:971-5.

58. Salem MK, Butt HZ, Watts AP, et al. Spontaneous cerebral embolisation in asymptomatic and recently symptomatic patients with TIA/Minor stroke. Eur J Vasc Endovasc Surg 2011;41:720-5.

59. Markus H, Cullinane M, et al. Severely impaired cerebrovascular reactivity predicts stroke and TIA risk in patients with carotid artery stenosis and occlusion. Brain 2001;124:457-67.

60. Gupta A, Chazen JL, Hartman M, et al. Cerebrovascular reserve and stroke risk in patients with carotid stenosis or occlusion: a systematic review and meta-analysis. Stroke 
2012;43:2884-91.

61. Reinhard M, Schwarzer G, Briel M, et al. Cerebrovascular reactivity predicts stroke in high-grade carotid artery disease. Neurology 2014;83:1424-31.

62. Kluytmans M, van der Grond J, van Everdingen KJ, et al. Cerebral hemodynamics in relation to patterns of collateral flow. Stroke 1999;30:1432-9.

63. Henderson RD, Eliasziw M, Fox AJ, et al. Angiographically defined collateral circulation and risk of stroke in patients with severe carotid artery stenosis. North American Symptomatic Carotid Endarterectomy Trial (NASCET) Group. Stroke 2000;31:128-32.

64. Warach SJ, Luby M, Albers GW, et al. Acute Stroke Imaging Research Roadmap III Imaging Selection and Outcomes in Acute Stroke Reperfusion Clinical Trials: Consensus Recommendations and Further Research Priorities. Stroke 2016;47:1389-98.

65. Vermeer SE, Longstreth WT Jr, Koudstaal PJ, et al. Silent brain infarcts: a systematic review. Lancet Neurol 2007;6:611-9.

66. Kakkos SK, Sabetai M, Tegos T, et al. Silent embolic infarcts on computed tomography brain scans and risk of ipsilateral hemispheric events in patients with asymptomatic internal carotid artery stenosis. J Vasc Surg 2009;49:902-9.

67. Baradaran H, Gialdini G, Mtui E, et al. Silent Brain Infarction in Patients With Asymptomatic Carotid Artery Atherosclerotic Disease. Stroke 2016;47:1368-70.

68. Gensicke H, van der Worp HB, Nederkoorn PJ, et al. Ischemic brain lesions after carotid artery stenting increase future cerebrovascular risk. J Am Coll Cardiol 2015;65:521-9.

69. Traenka C, Engelter ST, Brown MM, et al. Silent brain infarcts on diffusion-weighted imaging after carotid revascularisation: A surrogate outcome measure for procedural stroke? A systematic review and meta-analysis. Eur Stroke J 2019;4:127-43.

70. Debette S, Schilling S, Duperron MG, et al. Clinical Significance of Magnetic Resonance Imaging Markers of Vascular Brain Injury: A Systematic Review and Metaanalysis. JAMA Neurol 2019;76:81-94.

71. Wardlaw JM, Smith EE, Biessels GJ, et al. Neuroimaging standards for research into small vessel disease and its contribution to ageing and neurodegeneration. Lancet Neurol 2013;12:822-38.

72. Streifler JY, Eliasziw M, Benavente OR, et al. Prognostic importance of leukoaraiosis in patients with symptomatic internal carotid artery stenosis. Stroke 2002;33:1651-5.
73. Timmerman N, Rots ML, van Koeverden ID, et al. Cerebral Small Vessel Disease in Standard Pre-operative Imaging Reports Is Independently Associated with Increased Risk of Cardiovascular Death Following Carotid Endarterectomy. Eur J Vasc Endovasc Surg 2020;59:872-80.

74. Ederle J, Davagnanam I, van der Worp HB, et al. Effect of white-matter lesions on the risk of periprocedural stroke after carotid artery stenting versus endarterectomy in the International Carotid Stenting Study (ICSS): a prespecified analysis of data from a randomised trial. Lancet Neurol 2013;12:866-72.

75. Johri AM, Nambi V, Naqvi TZ, et al. Recommendations for the Assessment of Carotid Arterial Plaque by Ultrasound for the Characterization of Atherosclerosis and Evaluation of Cardiovascular Risk: From the American Society of Echocardiography. J Am Soc Echocardiogr 2020;33:917-33.

76. Saba L, Yuan C, Hatsukami TS, et al. Carotid Artery Wall Imaging: Perspective and Guidelines from the ASNR Vessel Wall Imaging Study Group and Expert Consensus Recommendations of the American Society of Neuroradiology. AJNR Am J Neuroradiol 2018;39:E9-E31.

77. Ridker PM, Everett BM, Thuren T, et al. Antiinflammatory Therapy with Canakinumab for Atherosclerotic Disease. N Engl J Med 2017;377:1119-31.

78. Tardif JC, Kouz S, Waters DD, et al. Efficacy and Safety of Low-Dose Colchicine after Myocardial Infarction. N Engl J Med 2019;381:2497-505.

79. Nidorf SM, Fiolet ATL, Mosterd A, et al. Colchicine in Patients with Chronic Coronary Disease. N Engl J Med 2020;383:1838-47.

80. Geurts L, Biessels GJ, Luijten P, et al. Better and faster velocity pulsatility assessment in cerebral white matter perforating arteries with 7T quantitative flow MRI through improved slice profile, acquisition scheme, and postprocessing. Magn Reson Med 2018;79:1473-82.

81. Timmerman N, Galyfos G, Sigala F, et al. The TAXINOMISIS Project: A multidisciplinary approach for the development of a new risk stratification model for patients with asymptomatic carotid artery stenosis. Eur J Clin Invest 2020;50:e13411.

82. Lal BK, Dux MC, Sikdar S, et al. Asymptomatic carotid stenosis is associated with cognitive impairment. J Vasc Surg 2017;66:1083-92.

83. Donahue MJ, Achten E, Cogswell PM, et al. Consensus statement on current and emerging methods for the 
diagnosis and evaluation of cerebrovascular disease. J

Cereb Blood Flow Metab 2018;38:1391-417.

84. Schröder J, Heinze M, Günther M, et al. Dynamics of brain perfusion and cognitive performance in revascularization of carotid artery stenosis. Neuroimage Clin 2019;22:101779.

Cite this article as: Donners SJA, Toorop RJ, de Kleijn DPV, de Borst GJ. A narrative review of plaque and brain imaging biomarkers for stroke risk stratification in patients with atherosclerotic carotid artery disease. Ann Transl Med 2021;9(15):1260. doi: 10.21037/atm-21-1166 